\title{
PALINOLOGÍA DE LOS GÉNEROS DE CACTACEAE MAIHUENIA (MAIHUENIOIDEAE) Y PERESKIA (PERESKIOIDEAE) DE ARGENTINA
}

\author{
GRACIELA A. CUADRADO ${ }^{1,2}$ \& SILVINA S. GARRALLA ${ }^{1,3}$
}

\begin{abstract}
Summary: Cuadrado G. A. \& S. S. Garralla. 2009. Palynology of the genera of Cactaceae Maihuenia (Maihuenioideae) and Pereskia (Pereskioideae) from Argentina. Bonplandia 18(1): 5-12. ISSN: 0524-0476.

Pollen grains of five species from two genera of the subfamilies Maihuenioideae and Pereskioideae (Cactaceae) are described with the optical and scanning microscopes. Maihuenia (F.A.C.Weber) K.Schum.: M. patagonica (Phil.) Britton \& Rose and M. poeppigii (Otto ex Pfeiff.) F.A.C. Weber ex K. Schum. (Maihuenioideae) and Pereskia Mill.: P. aculeata Mill., P. nemorosa Rojas Acosta and P. sacharosa Griseb. (Pereskioideae). The studied pollen grains of genera Maihuenia are prolate or spheroidal, medium or large sized, and Pereskia are all spheroidal and large sized. The apertures show variation in number: three to fourteen, colpate, with different pattern distribution and length of the colpus: Maihuenia has tricolpate grains and Pereskia 6-14 pantocolpate, some of them form syncolpos. The sculpture does not show marked differences between the species, which is microechinate and nanoechinate with perforations surrounded by a thickening. A key to determine the species of each genus is presented.
\end{abstract}

Key words: Pollen, morphology, taxonomy.

Resumen: Cuadrado, G. A. \& S. S. Garralla 2009. Palinología de los géneros de Cactaceae Maihuenia (Maihuenioideae) y Pereskia (Pereskioideae) de Argentina. Bonplandia 18(1): 5-12. ISSN: 0524-0476.

Fueron estudiados y descriptos con microscopio óptico y microscopio electrónico de barrido, los granos de polen de cinco especies argentinas de dos géneros, Maihuenia (F.C.C.Weber) K. Schum. y Pereskia Mill., pertenecientes a las subfamilias Maihuenioideae y Pereskioideae (Cactaceae). Maihuenia patagonica (Phil.) Britton \& Rose, M. poeppigii (Otto ex Pfeiff.) F.A.C. Weber ex K. Schum., (Maihuenioideae), Pereskia aculeata Mill., P. nemorosa Rojas Acosta y $P$. sacharosa Griseb. (Pereskioideae). Los granos de polen de Maihuenia son prolatos 0 esferoidales, medianos o grandes y los de Pereskia son todos esferoidales y grandes. Las aperturas muestran variación en número, tres a catorce, pero todas son colpadas y tienen diferente modelo de ubicación: Maihuenia tiene granos tricolpados y Pereskia 6-14 pantocolpados, algunos de éstos forman sincolpos. La escultura no muestra marcadas diferencias entre las especies, ella es microequinada y nanoequinada con perforaciones rodeadas de un engrosamiento. Se presenta una clave para diferenciar las especies de cada género.

Palabras clave: Polen, morfología, taxonomía.

${ }^{1}$ Asignatura Palinología, Facultad de Ciencias Exactas y Naturales y Agrimensura, UNNE. Centro de Ecología Aplicada del Litoral (CECOAL). Ruta 5 km 2,5 C.C. 291, (3400), Corrientes, Argentina.

${ }^{2}$ gracielacuadrado@hotmail.com

3 silgabe2@yahoo.com.ar 


\section{Introducción}

La mayoría de los géneros de Cactaceae citados para Argentina pertenecen a la subfamilia Cactoideae (22), siguen las Opuntiodeae (9) y con un género las Maihuenioideae y Pereskioideae respectivamente. Las especies de Maihuenioideae son de hábito subarbustivo suculento y se distribuyen en las provincias de Santa Cruz, Río Negro, Chubut, Neuquén y Mendoza, mientras que las de Pereskioideae son de hábito arbustivo suculento y arbustivo escandente, con representantes en las provincias de Catamarca, Chaco, Corrientes, Entre Ríos, Misiones, Formosa, Jujuy y Salta (Kiesling, 1999).

El objetivo de este trabajo es el estudio exhaustivo de la morfología polínica de las especies argentinas de los géneros Maihuenia (F.A.C.Weber) K. Schum. (Maihuenioideae) con dos especies: M. patagonica (Phil.) Britton \& Rose y M. poeppigii (Otto ex Pfeiff.) F.A.C. Weber ex K. Schum. y Pereskia Mill. (Pereskioideae) con tres especies: $P$. aculeata Mill., P. nemorosa Rojas Acosta y P. sacharosa Griseb. Una de las inquietudes que condujo a este estudio fue el hecho de haberse producido en Argentina, el primer hallazgo de polen fósil de Cactaceae, del Terciario (Mioceno Medio), proveniente de la Formación San José en la provincia de Tucumán (Mautino \& Anzótegui, 2002). Esta situación, puso de manifiesto la escasa bibliografía palinológica con que se cuenta sobre la familia en Argentina, necesaria para utilizarla en trabajos de polen disperso. No obstante es importante resaltar, los trabajos de Tsukada (1964) y Leuenberger (1976), quienes mencionan, entre otras, algunas especies de Argentina. Posteriormente, Leuenberger (1986) y (1997) publicó dos trabajos monográficos sobre las especies de Pereskia y de Maihuenia respectivamente, en donde analizó, entre otros caracteres, las generalidades del polen de ambos géneros. Recientemente, Garralla \& Cuadrado (2007) estudiaron palinológicamente las especies de Opuntioideae de Argentina, y Garralla \& al. 2008 las especies de Rebutia K. Schum. (Cactoideae) y sus implicancias en las definiciones de las especies de dicho género.
Por otra parte, dado que en los estudios de taxonomía se deben considerar la mayor cantidad posible de caracteres morfológicos para establecer relaciones entre especies, la morfología polínica aporta una información adicional y útil a la botánica, para clarificar diferencias y afinidades entre géneros o especies y para establecer un ordenamiento filogenético en una familia o taxones de mayor categoría. Esto se ve facilitado por el elevado número de especies de Cactaceae que habitan nuestro país, que según Kiesling (1999) es de 36 géneros y 225 especies.

\section{Materiales y Métodos}

Los ejemplares utilizados en este estudio provienen del Instituto de Botánica Darwinion (SI), Herbario Ruiz Leal (MERL) y el Instituto de Botánica del Nordeste (CTES) y la lista de especies para Argentina que abarca este trabajo fue tomada de Kiesling (1999).

Se analizó el polen de dos ejemplares de cada especie de acuerdo a la disponibilidad con que se contó. El material utilizado fue procesado con el método de acetólisis de Erdtman (1960), hidratados en agua glicerinada y montados en glicerina gelatina. Los preparados obtenidos, fueron depositados en la Palinoteca de la Universidad Nacional del Nordeste (PAL-CTES).

Para las observaciones y descripciones ópticas se utilizó un Microscopio Olympus BX 40 y las fotografías fueron tomadas con una cámara digital Canon Power Shot S55.

Las observaciones y descripciones mencionadas fueron complementadas con fotografías obtenidas con el Microscopio Electrónico de Barrido (MEB) Jeol/JSM- 580 LV de la Secretaria General de Ciencia y Técnica de la Universidad Nacional del Nordeste. El material utilizado para la obtención de estas fotografías también fue previamente acetolizado e hidratado en agua glicerinada.

Para establecer el tamaño de granos, espesor de la exina y el número y tamaño de aperturas, se realizaron observaciones y mediciones en 20 granos como mínimo, según los inconvenientes que presentó cada caso. 
Se utilizó la terminología sugerida por Erdtman (en Nilson \& Praglowski, 1992), consultándose también Punt \& al. (2007) para el nombre de las espinas de acuerdo a su distinta altura. Las descripciones sistemáticas se ordenaron alfabéticamente y se ilustraron todas las especies con fotografías de MO y MEB. El primer ejemplar citado en "Material estudiado" es el que se utilizó para la obtención de las mismas. No se ilustraron con MEB los detalles de la escultura de todas las especies debido a la similitud que presentaban. Las pequeñas diferencias en cuanto a las medidas de perforaciones, sus engrosamientos y altura de las espinas se observaron y midieron en el MEB y están mencionadas en todas las descripciones.

\section{Material estudiado}

Maihuenia patagonica (Phil.) Britton \& Rose. ARGENTINA. Mendoza: Dep. Malargüe, Ambrosetti \& del Vitto s. n. (MERL) Herbario Ruiz Leal 33475, PAL-CTES 7045. Leuenberger \& Arroyo 3805 (SI), PAL-CTES 7365.

Maihuenia poeppigii (Otto ex Pfeiff.) F.A.C. Weber ex K. Schum. ARGENTINA. Neuquén: Dep. Minas, Boelcke \& al. 10823 (SI), PALCTES 7046; Cordillera de Viento, Boelcke \& al. 11573 (SI), PAL-CTES 7047.

Pereskia aculeata Mill. ARGENTINA. Corrientes: Dep. Ituzaingó. Ea. Sta. Rita, en selva marginal del Rio Paraná. Krapovickas \& al. 41078 (CTES), PAL-CTES 7368. BRASIL. Paraná: Mun. Porto Rico, Kiesling 2195 (SI) PAL-CTES 7048.

Pereskia nemorosa Rojas Acosta. ARGENTINA. Corrientes: Dep. Capital, Tressens \& Schinini 3201 (SI), PAL-CTES 7366. PARAGUAY. San Pedro: Woolston 910 (SI) PALCTES 7053.

Pereskia sacharosa Griseb. ARGENTINA. Buenos Aires: Olivos, (cultivada) Stienstra 16298 (SI) PAL-CTES 7049. Formosa: Dep. Matacos, Fortunato 6746 (SI) PAL-CTES 7049. PARAGUAY. Chaco: Cerro León. Schinini \& Bordas 17813 (SI) PAL-CTES 7050.

\section{Resultados}

\section{Cacactaceae}

Subfamilia Maihuenioideae Maihuenia (F.A.C.Weber) K.Schum. M. patagonica (Phil.) Britton \& Rose

Fig. 1 A-F

Granos grandes, prolatos. Medidas: $\mathrm{P}=$ 52(58)62 $\mu \mathrm{m}, \mathrm{E}=37,5(39,6) 42 \mu \mathrm{m}$, ámbito circular, $\mathrm{P} / \mathrm{E}=1,4$. Tricolpados. Colpos largos, de aproximadamente $48 \mu \mathrm{m}$, apocolpios 10 $12 \mu \mathrm{m}$. Téctum cubierto por elementos positivos y negativos que no pueden definirse con el MO. Exina de $\pm 3 \mu \mathrm{m}$ de espesor. Sexina 1,5 $\mu \mathrm{m}$ y nexina $1,5 \mu \mathrm{m}$.

MEB: Esta observación determina que el téctum es microperforado con perforaciones de $0,13 \mu \mathrm{m}$ rodeadas de un engrosamiento anular de 0,38 $\mu \mathrm{m}$ (Fig.1 C, $6000 \mathrm{x}$ ) y nanoespinas supratectales de $\pm 0,5 \mu \mathrm{m}$ de altura. Se observan las membranas aperturales nanoequinadas en los colpos, con esculturas semejantes a las que cubren el resto del grano.

Distribución geográfica: Chubut, Mendoza, Neuquén, Río Negro, Santa Cruz.

\section{M. poeppigii (Otto ex Pfeiff.) F.A.C. Weber ex K. Schum.}

Fig. 1 G-L

Granos medianos, esferoidales. Medidas: 40(43,5)45,5 $\mu \mathrm{m}$. Tricolpados. Colpos largos, de aproximadamente $30 \mu \mathrm{m}$, apocolpios de \pm $10 \mu \mathrm{m}$. Téctum cubierto por elementos positivos y negativos que no pueden definirse. Exina de $\pm 2 \mu \mathrm{m}$ de espesor. Sexina $1,5 \mu \mathrm{m}$ y nexina $0,5 \mu \mathrm{m}$.

MEB: Esta observación determina que el téctum es microperforado con perforaciones de $0,2 \mu \mathrm{m}$, rodeadas de un engrosamiento de la misma medida que la perforación sólo distinguibles con altos aumentos (6000 x) y nanoespinas supratectales de $\pm 0,5 \mu \mathrm{m}$ de altura. Se observan las membranas aperturales en los colpos, con esculturas semejantes a las que cubren el resto del grano.

Distribución geográfica: Neuquén. 

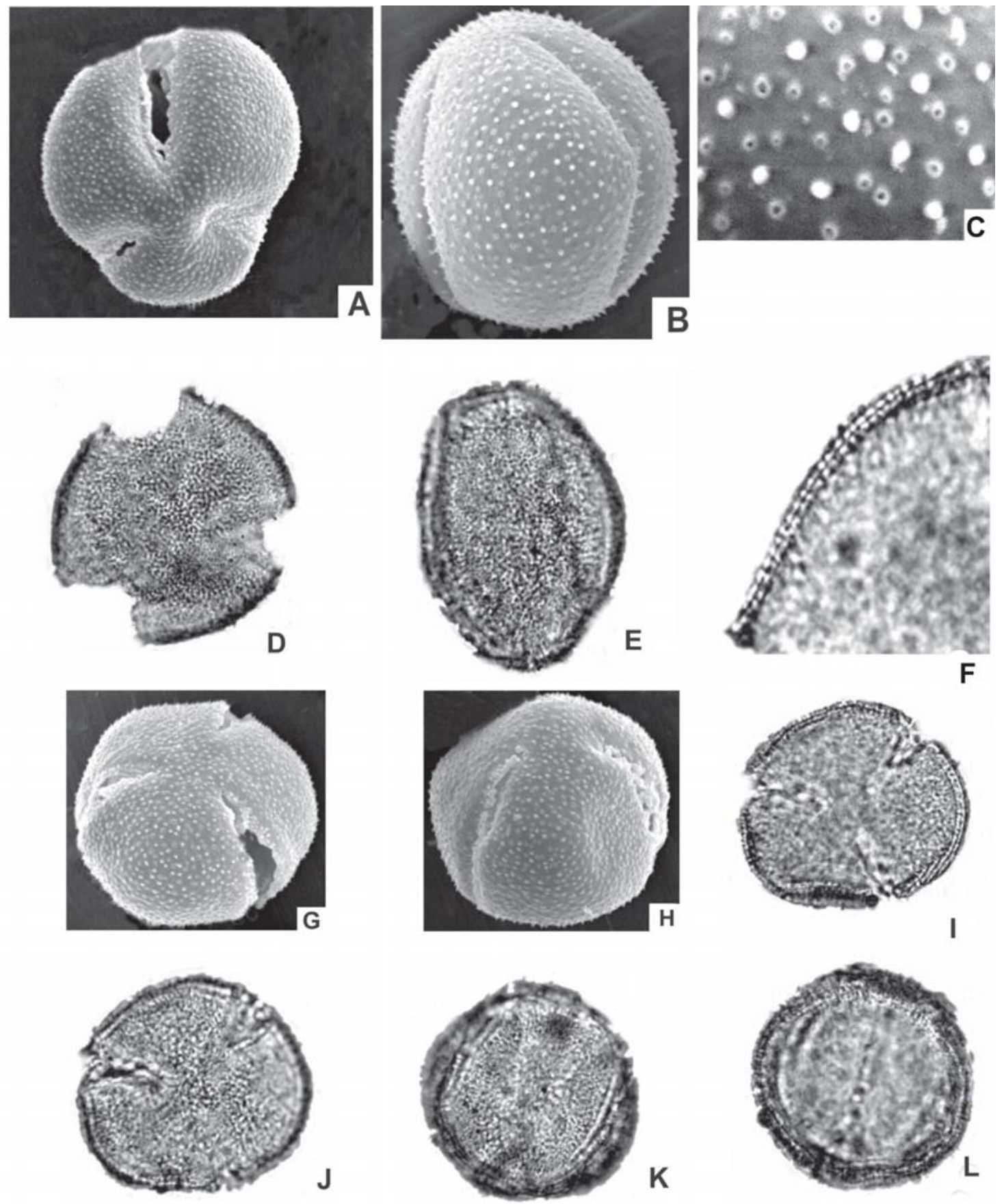

Fig. 1. Maihuenia patagonica. A: MEB, vista general polar. B: vista general ecuatorial. C: detalle de escultura. D: MO, vista general polar. E: vista general ecuatorial. F: detalle de corte óptico. (Ambrosetti \& Del Vitto s. n.). Mahuenia poeppigii G: MEB, vista general polar. H: vista general ecuatorial. I: MO, vista general polar, corte óptico. $\mathrm{J}$ : vista general polar, foco superior. K: vista general ecuatorial foco superior. L: vista general ecuatorial, corte óptico. (Boelke 10823). Escala = $20 \mu \mathrm{m}$ en A y D; $30 \mu \mathrm{m}$ en B y E; 7,6 en C; $30 \mu \mathrm{m}$, en F; $22 \mu \mathrm{m}$ en G y H y $28 \mu \mathrm{m}$ en I, J, K y L. 


\section{Pereskia Mill.}

\section{P. aculeata Mill.}

Fig. 2 A-E

Granos grandes, esferoidales. Medidas: 55(62,5)70 $\mu \mathrm{m}$. Pantocolpados, con $\pm 6-8$ colpos de aproximadamente $15-25 \mu \mathrm{m}$; en un $5 \%$ algunos colpos se reúnen formando un sincolpo. Téctum cubierto por elementos positivos y negativos muy pequeños cuya forma no puede determinarse por su tamaño, con el MO. Exina de $\pm 4 \mu \mathrm{m}$ de espesor. Sexina $3 \mu \mathrm{m}$ y nexina $1 \mu \mathrm{m}$.

MEB: Permite apreciar que el téctum es densamente perforado, estas perforaciones miden aproximadamente $0,3 \mu \mathrm{m}$ con engrosamientos anulares de $\pm 0,1 \mu \mathrm{m}$ (siguiendo el mismo esquema de las especies de Maihuenia) y nanoespinas supratectales de \pm 0,4 $\mu \mathrm{m}$ de altura (Fig. 2 B 4000x). Colpos con membranas aperturales esculturadas con nanoespinas, mas pequeñas que las que cubren la superficie del resto del grano.

Distribución geográfica: Corrientes, Entre Ríos, Misiones.

\section{P. nemorosa Rojas Acosta}

Fig. 2 F-H

Granos grandes, esferoidales. Medidas 50(55)60 $\mu \mathrm{m}$. Pantocolpado con 9-10 colpos de \pm 17 a $19 \mu \mathrm{m}$; en un $10 \%$ de los granos se reúnen formando un sincolpo. Téctum cubierto por elementos positivos y negativos los que por su tamaño no pueden definirse con el MO. Exina de $\pm 4 \mu \mathrm{m}$. Sexina $3 \mu \mathrm{m}$ y nexina $1 \mu \mathrm{m}$.

MEB: Puede observarse $(10.000 \mathrm{x})$ que el téctum tiene perforaciones que varían de diámetro: de $\pm 0,2$ a $0,7 \mu \mathrm{m}$ rodeadas de un engrosamiento anular que también varía de \pm 0,1 a $0,2 \mu \mathrm{m}(10000 \mathrm{x})$ la densidad de las perforaciones es mayor que en los casos anteriormente descriptos y microespinas de $\pm 0,95$ $\mu \mathrm{m}$ de altura. Colpos con membranas aperturales esculturadas con nanoespinas (Fig. 2 F).

Distribución geográfica: Corrientes, Entre Ríos, Misiones.

\section{P. sacharosa Griseb.}

Fig. 2 I-K

Granos grandes, esferoidales. Medidas 60(68)75 $\mu \mathrm{m}$. Pantocolpados, 12-14; colpos de 15 a $21 \mu \mathrm{m}$; en un $10 \%$ se reúnen formando dos sincolpos. Téctum cubierto por elementos positivos y negativos que no pueden discernirse con el MO. Exina de $\pm 3 \mu \mathrm{m}$ de espesor. Sexina $2 \mu \mathrm{m}$ y nexina $1 \mu \mathrm{m}$.

MEB: Se observan perforaciones de $\pm 0,2 \mathrm{a}$ $0,3 \mu \mathrm{m}$ con engrosamiento anular de $\pm 0,2 \mu \mathrm{m}$ a 0,5 y microespinas de $\pm 0,6 \mu \mathrm{m}$ de altura que siguen el mismo modelo de las especies anteriores. Se observan membranas aperturales ornamentadas con gránulos de $\pm 2,8 \mu \mathrm{m}$ de diámetro. No se hallaron diferencias entre la especie cultivada y las silvestres de Formosa y Paraguay.

Distribución geográfica: Chaco, Jujuy, Salta, Formosa, Catamarca.

\section{Clave de las especies argentinas de los géneros Maihuenia y Pereskia (Cactaceae)}

\section{Granos tricolpados}

2. Prolatos $(\mathrm{P} / \mathrm{E}=1,4)$, grandes

2'. Esferoidales $(\mathrm{P} / \mathrm{E}=1,1)$, medianos

M. patagonica

M. poeppigii 
1’. Granos pantocolpados

3. Colpos 6 a 8 , tectum con nanoespinas

3’. Colpos \pm 9 -14, tectum con microespinas

4. Colpos 9-10

4’. Colpos 12-14

\section{Discusión y Conclusiones}

Sobre la base de las observaciones realizadas al MO y al MEB los granos de polen de las especies argentinas pertenecientes a las subfamilias Maihuenioideae y Pereskioideae pueden diferenciarse por el número y disposición de las aperturas en dos grupos: granos tricolpados y granos pantocolpados. Entre los tricolpados se encuentran las especies del género Maihuenia ( $M$. patagonica y $M$. poeppigii) quienes a su vez pueden separarse por la forma y tamaño de sus granos y largo de los colpos. Por el contrario, Leuenberger (1997) sólo distingue a las dos especies por el número de espínulas en relación al número de perforaciones, no hallando otras diferencias significativas entre ellas. Los demás caracteres mencionados por el autor, coinciden con los observados en este trabajo, sólo que se refiere a ellos como comunes a Mahuenia como género y no como especies con algunas individualidades. En los pantocolpados están las especies de Pereskia donde se contaron colpos en número de 6 a 14, en tanto que Leuenberger (1976) contó una serie de 6-9-12 y 15 en Pereskias argentinas y Pereskia en general; es de destacar que en este género hay una importante variación en el largo de los colpos en un mismo grano; por otra parte entre un 5 y $10 \%$ de los granos, algunos colpos se reúnen formando uno o dos sincolpos (Figs. 2-B, E, H y K).

Tsukada (1964) analizó con MO 37 especies de Cactaceae, entre las cuales Pereskia aculeata difiere de la estudiada en este trabajo por presentar granos con mayor número de colpos. Heusser (1972), en su obra sobre el
P. aculeata

P. nemorosa

P. sacharosa

polen y las esporas de Chile describió con microscopio óptico el polen de Maihuenia poeppigii, cuyas características coinciden en su mayoría con la especie aquí analizada, sólo difiere en la forma de los granos, a quien el autor describe como subprolatos. Más tarde, Leuenberger (1976) estudió el polen de unas 600 especies de esta familia, de las cuales tres de ellas (Pereskia aculeata, P. sacharosa y Maihuenia poeppigii) son tratadas aquí, las características palinológicas brindadas por el autor, son similares a las arriba descriptas. En 1986, el mismo autor, realiza un estudio generalizado de la morfología del polen de Pereskia donde destaca "el amplio rango de medidas y número de aperturas” dentro del género; particularidad también observada en el polen de las especies argentinas.

Se ha encontrado una gran similitud entre Pereskia aculeata y $P$. nemorosa con Anredera cordifolia (Basellaceae) (Huk \& Pire, 2002), por el número de colpos, características de la exina e irregularidad en la disposición de los colpos, sólo que los granos de Anredera cordifolia son de un tamaño considerablemente menor a las Pereskias citadas.

Por otra parte, al comparar la morfología polínica de las especies correspondientes a las subfamilias estudiadas, con el polen de las otras Cactaceae de Argentina analizadas hasta el momento (Garralla \& Cuadrado, 2007; Garralla \& al., 2008; Salgado \& Garralla, 2008; Cuadrado \& Lattar, com. pers.), se desprende que Maihuenia patagonica presenta estrecha similitud en todos sus caracteres con Rebutia fabrisii var. aureiflora Rausch y Maihuenia poeppigii con $R$. minuscula subsp. minuscula K. Schum., de manera tal que resulta difícil diferenciarlas. También por el 

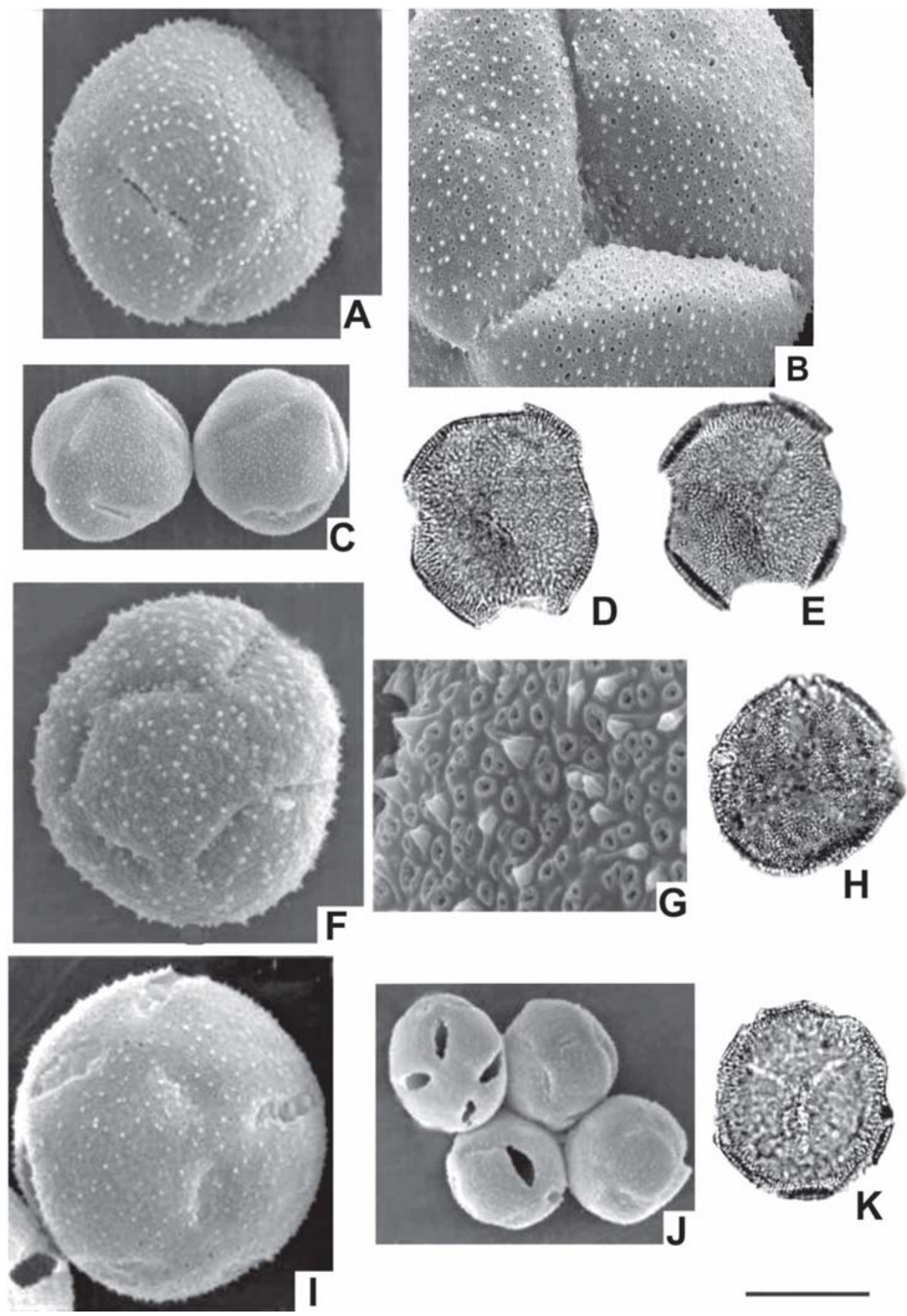

Fig. 2. Pereskia aculeata. A: MEB, vista general, mostrando escultura y los colpos en una vista. B: detalle de escultura en el áreaun sincolpo. C: dos granos de polen mostrando tres y cuatro colpos respectivamente. D: MO vista general corte óptico. E: vista general foco superior, mostrando tres colpos que forman un sincolpo (Kiesling 2195). Pereskia nemorosa. F: MEB, vista general mostrando escultura y 7 colpos. G: detalle de escultura. H: MO, vista general, corte óptico mostrando tres colpos que forman un sincolpo (Tressens \& Schinini 3201). Pereskia sacharosa. I: MEB, vista general mostrando escultura y 7 colpos. J: Cuatro granos mostrando los colpos en distintas posiciones. $\mathrm{K}$ : MO, vista general, mostrando dos sincolpos (Stienstra 16298). Escala $=25 \mu \mathrm{m}$ en A; $5 \mu \mathrm{m}$ en B; $50 \mu \mathrm{m}$ en C; 21,6 $\mu \mathrm{m}$ en D y E; $22 \mu \mathrm{m}$ en F; 4,6 $\mu \mathrm{m}$ en G; $36 \mu \mathrm{m}$ en H; 28 en I; $68 \mu \mathrm{m}$ en J y 46,6 en K. 
tipo de aperturas se asemejan palinológicamente a otros géneros de Cactoideae: Cereus Mill., Cleistocactus Lam., Denmoza Britton \& Rose, Echinopsis Zucc., Monvillea Britton \& Rose (tricolpados), Rebutia K. Schum. y Rhipsalis Gaertn. (tricolpados y pantocolpados).

Del estudio del polen de las especies argentinas de Pereskioideae y Maihuenoideae se desprende que sus características morfológicas son afines con las de la subfamilia Cactoideae, no así las Opuntioideae, como lo ya formulado por Leuenberger $(1986,1997)$.

\section{Agradecimientos}

Las autoras agradecen a los herbarios del Instituto Darwinion (SI), Ruiz Leal (MERL), e Instituto de Botánica del Nordeste (CTES) por proporcionar el material que hizo posible la realización de este trabajo.

A la técnica de Laboratorio Srta. Eva Acevedo y a las Técnicas del Microscopio Electrónico de Barrido (MEB), Lic. Cristina R. Salgado y Dra. Carolina Peichoto, por la cordialidad y buena disposición puesta en todo momento en la realización de sus tareas.

\section{Bibliografía}

ERDTMAN, G. 1960. The acetolysis method. Svensk. Bot. Tidskr. 54(4): 561-564.

GARRALlA, S. \& CUADRADO, G. 2007. Pollen morphology of the Cactaceae of Argentina (Opuntioideae): Austrocylindropuntia Backeb., Maihueniopsis Speg., Opuntia Mill. and
Tephrocactus Lem. Rev. Palaeobot. Palynol. 146: 1- 17.

GARRALLA, S., N. MURUAGA \& G. CUADRADO. 2008. Morfología polínica de especies argentinas de Rebutia s.str. (Cactaceae-Cactoideae). Darwiniana 46: 270-278.

HEUSSER, C. J.1971. Pollen and spores of Chile. The University of Arizona Press, Tucson. 167 pp.

HUK, L. H. \& S. M. PIRE. 2002. Basellaceae. En: S.M. Pire, L.M. Anzótegui \& G.A. Cuadrado (eds.). Flora Polínica del NEA 2: 47-50.

KIESLING, R. 1999. Cactaceae. En: F. O. Zuloaga \& O. Morrone (eds.), Catálogo de las Plantas Vasculares de la República Argentina 2. Monogr. Syst. Bot. Missouri Bot. Gard. 74: 423-489.

LEUENBERGER, B. E. 1976. Die pollenmorphologie der Cactaceae. Dissertationes Botanicae 31:1-321. 1986. Pereskia. Mem. New York. Bot. Gard. 41: $1-141$.

_- 1997.Mahuenia, monograph of a patagonian genus of Cactaceae. Bot. Jahrb. Syst. 119:1-92.

MAUTINO, L. R \& L. M. ANZÓTEGUI. 2002. Palinología de la Formación San José, Mioceno Medio, en la Provincia de Tucumán (Argentina). Resúmenes. VIII Congreso Argentino de Paleontología y Bioestratigrafía. Corrientes 7 al 10 de octubre de 2002.

NILSSON, S. \& J. PRAGLOWSKI. 1992. Erdtman's Handbook of Palynology. $2^{\circ}$. ed. Munksgaard, Copenhagen.

PUNT, W., HOEN, P. P., BLACKMORE, S. NILSSON, S. \& A. LE THOMAS. 2007. Glossary of pollen spore terminology. Rev. Palaeobot. Palynol. 143: 1-81.

SALGADO, C. R. \& S. S. GARRALLA. 2008. El polen de especies argentinas del género Rhipsalis Gaertn. (Cactaceae-Cactoideae). XIX Reunión de Comunicaciones Cientificas y Técnicas y Reunión de Extensión. Facultad de Ciencias Agrarias, Universidad Nacional del Nordeste. Corrientes, Argentina.

TSUKADA M., 1964. Pollen morphology and identifonation. II. Cactaceae. Pollen \& Spores 6(1): 45-84.

Original recibido el 21 de agosto de 2008; aceptado el 20 de octubre de 2008. 